\title{
Die Korrespondenz Duhem-Mach: Zur 'Modellbeladenheit' von Wissenschaftsgeschichte
}

\author{
Klaus Hentschel \\ Cankebeerstrasse 97, 2981 Westdorf, Bundesrepublik Deutschland
}

\author{
Received 27 March 1987
}

\begin{abstract}
Zusammenfassung
Die Korrespondenz der beiden Physiker und Wissenschaftshistoriker Ernst Mach und Pierre Duhem ist weitgehend, vielleicht mit Ausnahme nur eines Briefes, erhalten. Neben der Dokumentation dieser historischen Zeugnisse setzt sich der Autor in diesem Aufsatz zum Ziel, die jeweiligen Motive, die Mach resp. Duhem zur Beschäftigung mit Wissenschaftsgeschichte führten und die damit verbundenen Modelle der Wissenschaftsgeschichtsentwicklung beider gegeneinander abzugrenzen. Dazu wurden insb. die in der bisher vorliegenden Sekundärliteratur zu Mach und Duhem überhaupt nicht berücksichtigte Buchbesprechung der Machschen Mechanik durch Duhem sowie die ergänzenden Bemerkungen zu Duhem in späteren Auflagen der Werke und in der Korrespondenz Machs herangezogen, die eine recht detaillierte wechselseitige Kritik ihrer Methodik beinhalten. Den Abschluß meiner Betrachtungen bildet der Versuch einer Sondierung dessen, was in Duhems resp. Machs historischen Betrachtungsweisen über die nicht in Frage stehende Bedeutung als Meilensteine der Wissenschaftsgeschichtsschreibung hinaus noch heute von Relevanz ist.
\end{abstract}

\section{Summary}

The preserved part of the hitherto unpublished Duhem-Mach correspondence, kept in the Archives de l'Académie des Sciences, Paris, and in the Ernst Mach Institut of the Fraunhofer Society, Freiburg im Breisgau, is documented and commented upon. Biographical, methodological, and philosophical analogies and differences between the points of view of Pierre Duhem and Ernst Mach are discussed, and a critical evaluation is given of their impact as historians of science.

\section{Inhalt}

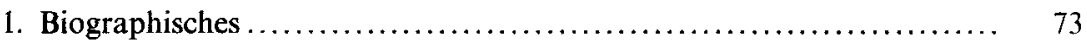

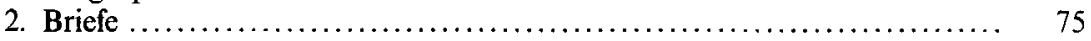

3. Duhems Auffassung von Ziel und Struktur physikalischer Theorien...... 82

4. Machs historisch-kritische Darstellung......................... 85

5. Machs Duhem-Replik und Duhems Mach-Besprechung: Differenzen der Auffassung und gegenseitige Kritik .............................. 86

5.1. Machs Bemerkungen zu Duhem ........................... 86

5.2. Duhem über Mach ........................................ 88

6. Zusammenfassende kritische Bewertung beider Auffassungen ............ 88

\section{Biographisches}

Pierre (Maurice Marie) Duhem (1861-1916) und Ernst (Waldfried Josef Wenzel) Mach (1838-1916) prägten die Wissenschaftsgeschichtsschreibung der Jahrhundertwende. Sie entfachten, wie im folgenden belegt wird im wesentlich unabhängig voneinander, jeder in seinem Einflußbereich ein erneutes Interesse an der zu ihrer Zeit nicht geschätzten historischen Betrachtung der Wissenschaft. 
Schon biographisch lassen sich weitere Parallelen hinzufügen:

(1) Beide waren ausgebildete Physiker, die schon mit ihren Erstlingsarbeiten das Aufsehen der Fachöffentlichkeit erregten, wodurch sie von Anbeginn ihrer wissenschaftlichen Laufbahn eine Sonder- bis Außenseiterstellung einnahmen. Mach nahm in einem seiner ersten Aufsätze 1860 Stellung in der Kontroverse zwischen Chr. Doppler und seinem akademischen Lehrer J. Petzval und verteidigte die Behandlung des später sog. Dopplereffektes gegen die ihm ungerechtfertigt scheinenden Angriffe seines Lehrers; eine Assistentenstelle blieb ihm in Wien daraufhin jedoch versagt. Duhem legte 1864 eine Promotion vor, in der er die Fehlerhaftigkeit des von Berthelot vorgeschlagenen Prinzips maximaler Arbeit nachwies, demzufolge die Reaktionsgeschwindigkeit chemischer Umsetzungen nur von der freiwerdenden Wärme abhing und entwickelte statt dessen eine (korrekte) Behandlung zahlreicher Phänomene der physikalischen Chemie (wie z.B. Kapillarität, Pyroelektrizität, osmotischer Druck, verallgemeinerte Gleichgewichtsbedingungen) durch konsequenten Bezug auf thermodynamische Potentiale. Dies wurde ihm von Berthelot und seinen Anhängern jedoch so verübelt, daß Duhem zeit seines Lebens nicht auf einen Lehrstuhl für theoretische Physik in Paris berufen wurde.

(2) Beide bewiesen ungewöhnliche Kreativität schon in ihren rein physikalischen Arbeiten. ${ }^{1}$ Duhem lehrte nach kurzen Lehrverpflichtungen in Lille und Rennes insg. 20 Jahre über theoretische Physik in Bordeaux ${ }^{2}$ mit Schwerpunkten in Thermodynamik, Physikalische Chemie, Hydromechanik und Elastizitätstheorie; ${ }^{3}$ Mach unternahm insb. in seiner Grazer und Prager Dozentenjahren experimentalphysikalische Untersuchungen (u.a. über den Dopplereffekt, Funkenwellen und Projektilphotographie) ebenso wie sinnesphysiologische Studien (z.B. über das Gleichgewichtsorgan und Sinnesphysiologie des Ohrs und Auges) und widmete sich nach Übernahme von Professuren für Physik in Prag (1876-95) bzw. für 'Philosophie, insb. Geschichte und Theorie der induktiven Wissenschaften' in Wien (1895-1901) theoretischen Untersuchungen (insb. zur Mechanik und zur Erkenntnistheorie). ${ }^{4}$

(3) Beide kamen im Verlauf ihrer Spezialstudien zu grundsätzlicher Kritik am überkommenen Denkstil in den Fachdisziplinen; im mechanistischen Weltbild sahen beide eine nicht gerechtfertigte Vorrangstellung der Mechanik, ${ }^{5}$ der Duhem eine verallgemeinerte Thermodynamik und Mach eine Physik, Physiologie und Psychologie umfassende Einheitswissenschaft entgegenzustellen versuchten.

\footnotetext{
${ }^{1}$ Bibliographien u.a. in Mémoires de la Société des Sciences Physiques et naturelles, Ser. 7, I, Teil 2 (1927) (Duhem) resp. in Centaurus, 8 (1963), S.189-237 (Mach).

${ }^{2}$ Zur Duhem-Biographie vgl. z.B. D. G. Miller, 'Duhem, Pierre-Maurice-Marie', in Dictionary of Scientific Biography, IV, 1971, S.225-233 sowie D. G. Miller, 'Ignored Intellect. Pierre Duhem', Physics Today, Dez. 1966, S.47-53; St. L. Jaki, Uneasy Genius. The Life and Work of Pierre Duhem (The Hague, 1984) (=International Archives of the History of Ideas, 100) sowie die dort zitierten Quellen.

${ }^{3}$ Vgl. neben D. G. Miller, a.a.O. auch F. Mentré, 'Pierre Duhem: le Théoricien', Revue de Philosophie, 22 (1922), $449-473$ u. 608-627.

${ }^{4}$ Zur Mach-Biographie vgl. u.a. J. T. Blackmore, Ernst Mach (Berkeley und Los Angeles, 1972; im folgenden zitiert als Blackmore, 1972) sowie K. Hentschel, 'Ernst Mach', Neue Deutsche Biographie, Bd.15, (1987), s. 605-609.

${ }^{5}$ Vgl. z.B. J. Heilbron, 'Duhem and Donahue'. Kommentar zu R. S. Westman, The Copernican Achievement (Los Angeles, 1975), S. 276-284, sowie z.B. Abschnitt 9 von Duhems Besprechung von Machs, Mechanik, zit. in Anm. 12.
} 
(4) Beide polemisierten gegen den Atomismus ihrer Zeit, wenn dieser nicht als Arbeitshypothese, sondern als Aussage über die reale Struktur der Materie verstanden wurde; letzteres schien beiden aufgrund der damaligen experimentellen Situation eine nicht gerechtfertigte Annahme. ${ }^{6}$ Beide arbeiteten deshalb zeit ihres Lebens im Rahmen von Kontinuumsphysik.

(5) Beide plädierten, wenngleich aus völlig unterschiedlichen Motiven heraus (s.u.), für eine strikte Trennung von Physik und Metaphysik.

(6) Beide verbanden ihre innerwissenschaftlich begründeten Neuorientierungsversuche mit wissenschaftshistorischer Betrachtung ihrer Disziplin, an der für sie eben nicht lediglich die Darstellung des status quo, sondern gerade auch die Genese der von ihnen immer als vorläufig betrachteten Resultate interessant war. Konsequenterweise gehörte für sie zur Erarbeitung einer Disziplin auch das Überschauen von deren Vergangenheit, weshalb sie, unabhängig voneinander, historisch-genetisch angelegte Abhandlungen verfassten. ${ }^{7}$

\section{Briefe}

$\mathrm{Da}$ die so geistesverwandten Denker erst 1903 voneinander Notiz genommen haben, ist zum einen erklärlich dadurch, daß der rund 20 Jahre jüngere Duhem erst nach der Jahrhundertwende, also nach Abschluß der 'historisch-kritischen Darstellungen' Machs, mit der Veröffentlichung größerer historisch angelegter Studien begann, zum anderen damit, daß die wechselseitige Rezeption deutsch- resp. französischsprachiger Literatur traditionell schlecht bestellt war, teils wegen Sprachbarrieren, ${ }^{8}$ teils wegen tiefverwurzelter Antipathien beider Völker. ${ }^{9}$

Erst als Machs wohl wichtigste historisch-kritische Darstellung, die der Mechamik, ${ }^{10}$ ins Französische übersetzt werden sollte, stieß Mach bei der Durchforstung der einschlägigen französischen Literatur auf Duhems parallele Bemühungen, wie er anläßlich des Erscheinens der 5. deutschen Auflage seiner Mechanik berichtete: ${ }^{11}$

Die kürzlich erschienene französische Ausgabe dieses Buches hat mir den Anlass geboten, die französische methodologische Literatur der Mechanik genauer anzusehen. Schriften wie jene von G. Lechalas (Étude sur l'espace et le temps, 1896), E. Picard (Quelques réflexions sur la mécanique, 1902), H. Poincaré (La science et l'hypothèse) und vor allem das von tiefem und umfassenden Blick zeugende Buch von P. Duhem (L'évolution de la mécanique, 1903) lassen mich hoffen, dass meine Schrift auch in Frankreich, wo naheliegende Wege schon eingeschlagen worden sind, freundliche Aufnahme finden wird.

\footnotetext{
${ }^{6} \mathrm{Vgl}$. dazu E. N. Hiebert, 'The genesis of Mach's early views on atomism', Boston Studies in the Philosophy of Science, 6 (1970), 79-106; J. T. Blackmore, 'An historical note on Ernst Mach', British Journal for the Philosophy of Science, 36 (1985), 299-329, und dort zitierte weitere Quellen.

${ }^{7}$ Mach eher disziplinenorientiert (Mechanik, Wärmelehre, Optik), Duhem eher ideengeschichtlich (Statik, chemische Verbindungen, Kosmologie in der Tradition des $\Sigma$ RZEIN TA ФAINOMENA).

${ }^{8} \mathrm{DaB}$ Mach des Französischen mächtig war, dürfte angesichts der vorwiegend humanistisch ausgebildeten Wissenschaftler seiner Generation eine Ausnahme gewesen sein.

${ }^{9}$ Vgl. z.B. A. Kleinert, 'Von der Science Allemande zur Deutschen Physik. Nationalismus und moderne Naturwissenschaft in Frankreich und Deutschland zwischen 1914 und 1940', Francia. Forschungen zur westeuropäischen Geschichte, 6 (1978), 509-25.

${ }^{10}$ Ernst Mach, Die Mechanik in ihrer Enwwicklung historisch-kritisch dargestellt (Leipzig, dt. Erstausgabc 1883!. 9. Aufl. 1933) [im folgenden zitiert als Mechanik].

"Ibid p. xiii, im Vorwort, datiert Wien März. 1904].
} 
Schon knapp ein Jahr vor Erscheinen des französischen Übersetzung sandte Mach, offensichtlich erfreut über den unerwarteten Mitstreiter, die 1903 erschienene 3. Auflage der Populär-wissenschaftlichen Vorlesungen an Duhem. Dieser reagierte darauf mit dem ersten erhaltenen Brief der Korrespondenz vom 9.7.1903 wie folgt:

Duhem an Mach, 9 Juli 1903 (handschr. Brief)

Université de Bordeaux

Faculté Des Sciences

Laboratoire de Physique Théorique

Bordeaux, le 9 juill. 1903.

Monsieur et très honoré Collègue,

J'ai reçu ce matin votre très aimable lettre et vos conférences populaires; de ce double envoi, permettez moi de vous remercier chaleureusement.

Je recevrai également avec grande joie la traduction française de votre mécanique; mais je n'en aurai pas la surprise; M. Hermann a eu l'amabilité de me la communiquer en épreuves; aussi ai je pu en faire une analyse étendue, qui sera insérée dans le Bulletin des Sciences Mathématiques, qui est déjà composée, et qui paraîtra peu après l'ouvrage même. Je me suis efforcé de faire partager au lecteur l'extrême intérêt que j'ai pris à lecture de votre livre; j'espère, en tous cas, que vous $\mathrm{y}$ verrez un témoignage de mon admiration pour votre oeuvre.

Cette lecture m'a montré que dans plusieurs circonstances j'avais retrouvé des idées que vous aviez écrites depuis longtemps et que je les avais publiées sans vous citer; je profiterai de toutes les occasions qui se présenteront pour réparer mon omission et vous rendre la justice qui vous avez due.

Croyez, Monsieur et très Honoré Collègue, à mon très respectueuz dévouement. (P. Duhem)

Dieser Brief zeigt, daß auch Duhem sofort die Geistesverwandtschaft zu Mach erkannte. Schon die ganz ungewöhnliche Länge seiner Buchbesprechung ${ }^{12}$ (vgl. Abschnitt 5 b dieses Aufsatzes) zeugt von der Intensität, mit der Mach ihn beeindruckt haben muB: die elf Abschnitte auf insg. 22 Seiten erschienen, auch dies wohl ein Unikum in der Wissenschaftsgeschichte, noch vor Erscheinen des Buches selber (1904) bereits im Jahr 1903, hastig in den letzten Teil des im Brief zitierten Bulletins eingefügt. Daß dies überhaupt so schnell vonstatten ging, war wohl auch dem Umstand zu verdanken, daß einer der Herausgeber des Bulletins, Emile Picard (1856-1941) auch das Vorwort zur französischen Übersetzung der Mechanik verfasst hatte.

Schon zu Beginn des nächsten Jahres wiederholte Mach die freundschaftliche Geste mit der Übersendung der 2. Auflage seiner Prinzipien der Wärmelehre. ${ }^{13}$ Besonders im neu hinzugekommenen Kapitel 'Der Substanzbegriff' ${ }^{14}$ und der darin klar ausgesprochenen Stellungnahme gegen Boltzmann ${ }^{15}$ dürfte Duhem aufs Neue die

\footnotetext{
${ }^{12}$ Ohne Titel, Bulletin des Sciences Mathématiques, 2. Serie, 27 (1903), 261-283 [im folgenden zitiert als Bulletin].

${ }^{13}$ E. Mach, Prinzipien der Wärmelehre. historisch-kritisch entwickelt (Leipzig, 1900).

14 2. Auflage, S. 423-431.

${ }^{15}$ Mach schrieb dort [S. 430f.]: 'Ich habe daher auch gar nichts dagegen zu sagen, wenn Boltzmann die Vorzüge preist, welche die Atomtheorie für den Physiker vor allen anderen Auffassungen voraus hat. Der Forscher darf nicht nur, sondern soll alle Mittel verwenden, welche ihm helfen können. Nur gegen das dauernde Festhalten willkürlicher Zuthaten zu den Thatsachen spreche ich.... Ich kann darum auch nicht einsehen, warum jede Differentialgleichung sich nothwendig auf atomistische Betrachtungen gründen müsste."
} 
Nähe ihrer beiden Positionen erkannt haben. Duhem reagierte wie folgt:

Duhem an Mach, 3. März 1904 (handschriftl, Karte)

Monsieur et très honoré Collègue,

Bordeaux, 3/3.04.

Permettez moi de vous remercier très vivement de votre ouvrage sur la Thermodynamique, dont je viens de recevoir la nouvelle édition, il m'intéresse doublement, et par le sujet traités et par la personne de l'auteur.

Croyez à mes sentiments respectueusement dévoués.

(P. Duhem)

Mach reagierte diesmal nur sehr knapp.

Mach an Duhem, 28. März 1904 (maschinenschr. auf Visitenkarte)

Monsieur et très cher Collègue,

excusez par une indisposition le retard de mes remerci[e]ments cordiales[sic.] pour votre aimable envoi.

28/III 1904

Votre dévoué

Der Plural, mit dem sich Duhem Ende April d.J. für Machs Briefe bedankt, läßt darauf schließen, daß Mach Anfang April noch einen ausführlicheren(?) nicht erhaltenen Brief an Duhem schrieb.

Duhem an Mach, 21 April 1904 (handschr. Brief)

Université de Bordeaux

Faculté Des Sciences

Laboratoire de Physique Théorique

Bordeaux, le 21 avril 1904.

Monsieur et cher Collègue,

J'aurais désiré, depuis longtemps, répondre à vos aimables lettres. J'attendais pour le faire, que l'imprimerie Gauthier Villars m'envoyât des bonnes feuilles de l'article que j'ai écrit sur votre beau livre; vous m'aviez dit, en effet, que vous n'aviez pu prendre connaissance de cet article. Je suis enfin en possession de ces bonnes feuilles, et je m'empresse de vous adresser [sic]. Je vous prie d'y voir une marque de mes sentiments d'admiration pour votre oeuvre.

Je suis confus que vous ayiez hésité à m'écrire en Allemand. De mon côté, en effet, je lis aisément l'Allemand, mais je ne suis pas int[?] assez sûr de mes connaissances pour oser l'écrire; aussi me suis-je permis de vous écrire en français.

Croyez, Monsieur et cher Collègue, à mes sentiments de respectueuz dévouement.

(P. Duhem)

Was die Zusendungen betrifft, die Mach im vorigen und folgenden Brief von Duhem in Antwort auf seine Buchübersendungen erhielt, lassen sich Mutmaßungen anhand des Verzeichnisses der Bibliothek Ernst Machs aufstellen. ${ }^{16}$ Dieses führt lediglich ein in Frage kommendes Buch Duhems 'mit eigenhändiger Widmung des

\footnotetext{
${ }^{16}$ Antiquariat Ackermann, Bibliothek Ernst Mach (2 Teile); Nr. 634 u. 636 (München 1959).
} 
Verfassers' auf, und zwar: L'évolution de la mécanique, Paris, 1903. ${ }^{17}$ Als Replik auf Machs Thermodynamik erscheint es plausibel, daß Duhem die ebenfalls von Mach besessenen Chemie-historischen resp. -pädagogischen Werke Le mixte et la combinaison chimique. Essai sur l'évolution d'une idée, Paris, 1902, und Thermodynamique et chimie. Leçons élémentaires à l'usage des chimistes, Paris 1902, ${ }^{18}$ gehandelt hat.

Mach an Duhem, 15. Mai 1904 (maschinenschr. Brief)

Hochgeehrter Herr College!

Ich war vier Wochen krank, und bin noch so geschwächt, dass ich thnen nur mit wenigen Zeilen für Ihren freundlichen Brief und für Ihre gütige Zusendung danken kann. Das "Bulletin" ist in Wien nur in einem Exemplar vorhanden, und es hat deshalb lange gedauert, bevor ich von Ihrem wohlwollenden Artikel Kenntnis nehmen konnte. Ich hatte denselben noch nicht lange gelesen, als der Abdruck ankam. Auch für die Andeutungen über die Verbesserung des Buches bin ich Ihnen sehr zu Dank verpflichtet.

In vorzüglicher Hochachtung

Wien $15 / \mathrm{V} 1904$

Ihr ergebenster (Dr Ernst Mach)

Diese 'Andeutungen' Duhems werden im Abschnitt 5 im Zusammenhang besprochen. Nachdem Mach Duhems Buchbesprechung gesehen hatte, war er offensichtlich an einer Intensivierung ihrer Beziehungen interessiert - er tastete mit der Übersendung seines epistemologischen Hauptwerkes Analyse der Empfindungen vor, wie weit die Parallelen der Auffassung ins Grundlegend-Philosophische hineinreichten.

Mach an Duhem, 11. Juli 1904 (maschinenschr. Brief)

Hochgeehrter Herr College!

Einige philosophische Bemerkungen zu Ihrer Freundlichen [sic] Analyse meiner Schrift über Mechanik ermuthigen mich, Ihnen meine "Analyse der Empfindungen" zusenden $\mathrm{zu}$ lassen. Ich habe dort die allgemeinen Gesichtspunkte, von denen ich ausgehe, etwas ausführlicher dargelegt, als in der Mechanik. Ich hoffe, dass Ihnen diese Schrift nicht zu sehr missfallen wird. Wie ich aus dem 2. Circular des Genfer Philosophencongresses ersehe, welchen ich wegen meiner alten Hemiplegie leider nicht besuchen kann, werden Sie dahin

kommen. Es thut mir nun doppelt leid, nicht theilnehmen zu können.

Mit den besten Wünschen für die Ferien,

in besonderer Hochachtung

Ihr ergebenster

Wien 11/VII 1904

(Dr Ernst Mach)

\footnotetext{
${ }^{17}$ Ibid., Nr. 665.

I Ihid.. Nr. 975 und 976.
} 
Duhem an Mach, 16. Juli 1904 (handschr. Karte)

Monsieur et cher Collègue,

Bordeaux, 16 juillet 1904.

J'ai reçu hier, en même temps que votre aimable lettre, votre Theorie der Empfindungen; je vous remercie vivement de l'une et de l'autre. Je vais, aussitôt que les vacances me le permettront, m'attacher à la lecture de votre livre, je suis assuré d'avance d'y prendre le plus vif intérêt.

J'ai envoyé un travail au Congrès de Genève; mais je ne pourrai mal heureusement m'y rendre en personne, mes devoirs de famille ne me permettent guère de voyager.

Croyez moi votre respectueusement dévoué.

\section{(P. Duhem)}

Der erwähnte Kongreß war der 2. Internationale Kongreß für Philosophie in Genf vom 4. 8. Sept. 1904, zu dem Duhem eine ca. 50 seitige historische Arbeit über die con einer konstanten Kraft erzeugte Beschleunigung einreichte. ${ }^{19}$ Nach dieser unmittelbaren Danksagung ohne inhaltlichen Kommentar wäre es an Duhem gewesen, durch Kritik oder Zustimmung den Kontakt zu intensivieren. Daß dies nicht erfolgte, hat neben der intensiven Arbeit Duhems an seinen monumentalen historischen Studien auch daran gelegen, daß er Machs phänomenalistischen Ansatz nicht teilte (vgl. Abschnitt 5).

Tatsächlich lebte der Kontakt erst Ende 1906 wieder auf, als Mach in einem neuen Anlauf die 2. Auflage seines wissenschaftspsychologischen Werkes Erkenntnis und Irrtum übersandte, in dessen 'Vorwort zur zweiten Auflage' er folgendes über Duhem und dessen gerade erschienenes wissenschaftstheoretisches Hauptwerk geschrieben hatte:

Sehr erfreut war ich durch Duhems Werk La théorie physique, son objet et sa structure (1906). ${ }^{20}$ So weit gehende Übereinstimmung hoffte ich bei Physikern noch nicht zu finden. Duhem weist jede metaphysische Auffassung physikalischer Fragen ab; er sieht in der begrifflich-ökonomischen Fixierung des Tatsächlichen das Ziel der Physik; er hält die historisch-genetisch Darstellung der Theorien für die einzig richtige und didaktisch zweckmäßige. Das sind Ansichten, die ich in Bezug auf Physik seit reichlich drei Decennien vertrete. Die Übereinstimmung ist mir um so wertvoller, als Duhem ganz unabhängig zu denselben Ergebnissen gelangt ist. Während ich aber, wenigstens, in dem vorliegenden Buch, hauptsächlich die Verwandtschaft des vulgären und des wissenschaftlichen Denkens hervorhebe, beleuchtet Duhem besonders die Unterschiede des vulgären und des kritisch-physikalischen Betrachtens und Denkens, so daß ich sein Buch meinen Lesern als ergänzende und aufklärende Lektüre wärmstens empfehlen möchte. In dem Folgenden werden ich oft auf Duhems Äußerungen zu verweisen und nur selten, in untergeordneten Punkten, eine Meinungsdifferenz zu bemerken haben.

\footnotetext{
${ }^{19} \mathrm{P}$. Duhem, 'De l'accélération produite par une force constante. Notes pour servir à l'histoire de la dynamique', Congrès Internationale de Philosophie. Rapports et Comptes Rendues (Genf 1905), S. 859-915.

${ }^{20}$ Französische Erstausgabe; die deutsche Übersetzung von Friedrich Adler mit einem Vorwort Machs erschien bereits 2 Jahre später: Ziel und Struktur der physikalischen Theorien (Leipzig, 1908; Reprint mit Finleitung u. Bibliographic v. L. Schäfer, Hamburg, 1978) [im folgenden zitiert als Ziel].
} 
Daß Mach in seinen Ausführungen über 'Unterschiede' wichtige Differenzen unter den Tisch gekehrt hatte und den vergleichenden Leser auf einen eher unerheblichen Seitenpfad lenkte, sei hier nur am Rande erwähnt und wird in Abschnitt 5 belegt.

Duhem freute sich selbstverständlich über Machs lobende Erwähnung:

Duhem an Mach, 4. Oktober 1906 (handschr. Brief)

Université de Bordeaux

Faculté Des Sciences

Laboratoire de Physique Théorique

Bordeaux, le 4 Oct. 1906.

Monsieur et très honoré Collègue,

En rentrant hier de vacances, j'ai trouvé le très gracieux hommage de la Seconde édition de votre beau livre: Erkenntniss und Irrthum; le fait que la première édition a été si rapidement épuisée dit bien haut l'intérêt qu'excite un pareil livre.

Je me suis empressé d'y jeter les yeux, et j'ai été à la fois très heureux et très confus de la préface, si aimable à mon égard, que vous y avez mise. Je n'aurais pu souhaiter, en faveur de mon modeste ouvrage, suffrage plus flatteur et plus autorisé que le vôtre; et l'accord de mes méditations avec les vôtres est, pour moi, l'une des meilleures marques que je me trouve dans la bonne voie.

Veuillez agréer, avec l'expression de ma vive reconnaissance, l'hommage de mon profond respect.

\section{(P. Duhem)}

In seinem Antwortbrief bezog Mach auch die andere der erwähnten Schriften Duhems Über die Ursprünge der Statik ${ }^{21}$ mit ein. Die 6. Auflage der deutschsprachigen Version der Mechanik Machs berücksichtigte diese 'Belehrungen' in einem 'Zusatz 5 [s.u.]; allerdings erschienen diese Bemerkungen erst 2 Jahre später (1906). ${ }^{22}$

Bemerkenswert an den Oktoberbriefen 1906 ist insb. die Übereinstimmung in ihrer Wertschätzung der Person Boltzmanns, die angesichts der moderaten und differenzierten Kritik an den wissenschaftlichen Auffassungen Boltzmanns, die ich oben zitierte, glaubhaft ist. Beide bekundeten diese Einschätzung auch in reinphysikalischen Beiträgen zur Boltzmann-Festschrift, die noch vor dessen Selbstmord (1906) zusammengestellt wurde. ${ }^{23}$

${ }^{21}$ Les Origines de la Statique (Paris, Vol. I; 1905, II, 1906) [im folgenden zitiert als Origines]; verzeichnet im Katalog Ackermann Nr. 667 (Band I) 'Einige Rotstiftanstreichungen'.

${ }^{22}$ Vgl. Mechanik, 6. Auflage 1908, S. 567: 'Aus Duhems historischer Darlegung geht ferner hervor, dass Descartes sich unabhängig von Galilei bedeutende Verdienste um die Entwickelung der Grundvorstellungen der modernen Dynamik erworben hat, als gewöhnlich angenommen wird und also auch ich angenommen habe... Ich bin für diese Belehrung aufrichtig dankbar.... [Descartes]erkannte, wie aus 1629, vor Galileis Publikation an Mersenne geschriebenen Briefen hervorgeht, vollständig das Trägheitsgesetz, das Gesetz der gleichförmig beschleunigten Bewegung unter dem Einfluß einer konstanten Kraft, und irrte nur in Bezug auf das Abhängigkeitsgesetz des Weges von der Zeit. Galileis und Descartes Gedanken ergänzen sich gegenseitig'...(vgl. Anm. 10).

${ }^{23}$ Festschrift Ludwig Boltzmann gewidmet zum 60. Geburtstage 20. Febr. 1904 (Leipzig, 1904); darin: P. Duhem, 'Sur la Stabilité éléctrique d'un milieu homogène et illimité', S. 13-27 und E. Mach, 'Objektive Darstellung der Interferenz des polarisierten Lichtes', S. 441-7. 
Mach an Duhem, 8. Oktober 1906 (maschinenschr. Brief)

Hochgeehrter Herr College!

Indem ich für Ihren gütigen Brief vom $4 / X$ herzlich danke, mache ich zugleich von Ihrer gütigen Erlaubnis Gebrauch, Ihnen deutsch zu schreiben.

Selten hat mich etwas so erfreut, wie die vielfache Uebereinstimmung mit Ihnen, und in so wichtigen Punkten, die sich mir durch Ihr herrliches Buch $L a$ théorie physique geoffenbart hat. Auch aus Les origines de la statique habe ich viel Belehrung geschöpft, namentlich haben Sie mich über das Verhältnis von Descartes zu Galilei aufgeklärt, wofür ich Ihnen sehr dankbar bin. Die 6. Ausgabe meiner Mechanik, welche Ihnen demnächst zugesendet werden soll, wird hiervon Zeugnis geben.

An dem tragischen Schicksal von P. J. Curie haben wir hier in Wien herzlichen Anteil genommen, und nun sind auch wir durch den Tod Boltzmanns, den Sie ja hochschätzen, schwer getroffen worden.

Mit den besten Wünschen, in aufrichtiger Verehrung Ihr ergebenster Wien 8/X'06.

(Dr Ernst Mach)

Duhem an Mach, 15. Oktober 1906 (handschr. Brief)

Université de Bordeaux

Faculté Des Sciences

Laboratoire de Physique Théorique

Monsieur et très honoré Collègue,

Bordeaux, 15 octobre 1906.

J'ai encore à vous remercier vivement et pour votre aimable lettre, et pour vos nouveaux envois.

Je suis très heureux et très flatté de l'intérêt que vouz voulez bien prendre aux Origines de la Statique; j'espére vous envoyer dans une quinzaine de jours le second volume de cet ouvrage. Je suis fort peiné de la mort de Boltzmann. Bien que ses préférences le portassent vers les théories atomiques et cinétiques que je n'aime guère, j'avais la plus grande admiration pour son beau talent. J'avais été très heureux de collaborer au volume qui lui fut offert pour son jubilé. C'est une grande perte pour la Physique mathématique.

Croyez, Monsieur et très honoré Collèque, à mes sentiments très respectueux. (P. Duhem)

Am Jahresende 1906 bedankte sich Mach für die Übermittlung zweier neuer Bücher durch den französischen Verleger Duhems:

Mach an Duhem, 29. Dezember 1906 (maschinenschr. Brief)

Hochgeehrter Herr College!

Gestern erhielt ich durch Herrn A. Hermann Ihre zwei neuen Schriften: Les Origines de la Statique II und Étude sur Léonard de Vinci, für welche ich Ihnen herzlichst danke. Ich habe bisher nur einen Blick hinein tun können, aber sogleich gesehen, welche Fülle des Belehrenden diese Schriften enthalten. Ihre Arbeitskraft ist wirklich zu bewundern, da Ihnen dieselbe gestattet neben Ihren theoretischen Arbeiten noch diese Menge schwieriger historischer Untersuchungen zu bewältigen. 
Nochmals dankend mit den besten Wünschen, in Verehrung Ihr Wien 29/XII '06

(Dr Ernst Mach)

Der nächste Kontakt beider ist erst einenhalb Jahre später nachweisbar und wieder an die wechselseitige Übersendung von neuen Werken gekoppelt.

Duhem an Mach, 8. April 1908 (handschr. Postkarte)

Bordeaux, 8 avril 1908.

Cher et Vénéré Collègue,

Je vous remercie vivement de m'avoir envoyé la nouvelle édition de votre Mécanique. J'applaudis du fond du coeur au succès toujours plus grand de votre belle oeuvre. Croyez, je vous prie, à mes sentiments très respecteux.

(P. Duhem)

Duhem an Mach, 10. August 1909 (handschr. Postkarte)

Bordeaux 10 août 1909

Monsieur et Vénéré Collègue,

Je vous félicite grandement d'avoir fait rééditer votre travail si important, et aujourd'hui introuvable, sur l'histoire de la conservation du travail, et je vous remercie du fond du coeur de m'en avoir adressé un exemplaire. Cet écrit peut, avec celui de Rankine sur l'Energétique, être regardé comme le point de départ de tout le mouvement épistémologique si actif aujourd'hui et que vos autres écrits ont tant contribué à propager.

Croyez, je vouz prie, à mon profond respect et permettez moi de me dire votre disciple.

$$
\text { (P. Duhem) }
$$

Spätere Korrespondenz hat sich nicht erhalten; jedoch verzeichnet der Katalog Ackermann unter Nr.230 un document relatif à la réforme du calendrier, Paris 1911 'mit eigenhändiger Widmung d. Verfassers.'

Es ist erstaunlich, daß Duhem und Mach in ihrer Korrespondenz über den Austausch von Höflichkeitsbezeugungen nicht hinausgekommen sind. Aus der Analyse von Duhems und Machs veröffentlichten Kommentaren in Buchbesprechungen bzw. späteren Ergänzungen von Werken lassen sich die in der Korrespondenz nicht ausgetragenen Meinungsverschiedenheiten deutlich machen, die letztlich erklären, wieso ihr Gedankenaustausch nicht informeller und ergiebiger wurde.

\section{Duhems Auffassung von Ziel und Struktur physikalischer Theorien}

Duhems Wissenschaftsgeschichtskonzept muß ebenso wie bei Mach in Zusammenhang mit seinen fachwissenschaftlichen Arbeiten gesehen werden. ${ }^{24}$ Es bildete sich heraus in Abgrenzung zu den Ansprüchen der aufkommenden Schule derjenigen, die wie z.B. J. Cl. Maxwell oder W. Thomson, in atomistischen Modellen der Materie einer Erklärung der beobachteten Phänomene sehen wollten. Nach

${ }^{24} \mathrm{Vgl}$. z.B. A. Rey, 'La philosophie scientifique de M. Duhem', Revue de métaphysique et de morale, 12 (1904), 699-744; A. Lowinger, The Methodology of Pierre Duhem (New York, 1967). 
Duhem waren solche 'Erklärungen' wertlos, da sie nur durch die Einführung von Annahmen möglich waren, die selbst 'unerklärt' bleiben mußten, sofern sich die jeweils unterlegten Modelle (in)elastischer Kugeln/Hanteln etc. nicht ohnehin untereinander widersprachen. Seiner Ansicht nach mußte jedwede physikalische Theorie unabhängig von meta-physischen Voraussetzungen formuliert werden, die nicht in ihr begründet werden konnten.

Eine physikalische Theorie ist keine Erklärung. Sie ist ein System mathematischer Lehrsätze, die aus einer kleinen Zahl von Prinzipien abgeleitet werden und den Zweck haben, eine zusammengehörige Gruppe experimenteller Gesetze ebenso einfach, wie vollständig und genau darzustellen. ${ }^{25}$

Dabei ist wesentlich, daß Duhem hier von 'System' spricht, in dem die einzelnen Lehrsätze stehen. Die Darstellung experimenteller Sachverhalte wird nicht als Zuordnung einzelner Tatsachen zu einzelnen Sätzen aufgefasst, sondern als eine Verdichtung vieler Einzeltatsachen in Theorien. Dabei ist der Zusammenhang von Beobachtungen und ihrer wissenschaftlichen Auswertung ein sehr vermittelter:

Zwischen den bei der Ausführung eines Experimentes wirklich festgestellten Erscheinungen und dem Resultat dieses Experimentes, das vom Physiker formuliert wird, muß eine intellektuelle, sehr komplizierte Arbeit eingeschaltet werden, die einen Bericht über die konkreten Tatsachen durch ein abstraktes und symbolisches Urteil substituiert. ${ }^{26}$

In die Konzeption des Experimentes, in die dabei benutzten Hilfsmittel und die als selbstverständlich vorausgesetzten Hilfshypothesen geht bereits Theorie ein [Theorienbeladenheit des Experimentes]. ${ }^{27}$ Ferner ist die Zuordnung von 'praktischen Tatsachen mit ihren Konturen, die so verschwommen und unbestimmt [sind], wie alles, was uns durch unsere Wahrnehmung kund wird', zu 'theoretischen Tatsachen, die genau und streng sind, ${ }^{28}$ selbst noch mit Konventionen der Abgrenzung von Phänomengruppen und der Symbolzuordnung überfrachtet [Konventionalismus]. Sowenig es auf der Beobachtungsebene möglich ist, einzelne Eigenschaften eines Körpers von anderen abzutrennen, ${ }^{29}$ sowenig können, Duhem zufolge, einzelne Theoreme 'individuell' getestet werden: sie wirken zusammen in einem netzartigen Gefüge, das durch jedes Experiment als Ganzes, quasi als Organismus geprüft wird [Holismus]. ${ }^{30}$

Entgegen den zeitgenössischen Vorstellungen, ${ }^{31}$ die noch in den Verifikationismus bzw. Falsifikationismus des Wiener Kreises bzw. Poppers ausstrahlter, bestritt Duhem vor dem Hintergrund seines Holismus die isolierte Testbarkeit einzelner Hypothesen ${ }^{32}$ Für den Vergleich zweier konkurrierender Hypothesen (etwa Korpuskularoptik Newtons versus Wellenoptik Huygens) implizierte dies die Ablehnung der seit Bacon behaupteten Möglichkeit eines 'experimentum crucis' ${ }^{33}$ Schon kleine Veränderungen

${ }^{25}$ Ziel (Anm. 20), S. 21.

${ }^{26}$ Ziel (Anm. 20), S. 200.

${ }^{27}$ Vgl. Duhems Diskussion der Lupe, des Mikroskops und der Tangentenbussole als Beispiele für verschiedene Stadien.

${ }^{28}$ Ziel (Anm. 20), S. 200.

${ }^{29}$ Jeder elektrisch geladene Körper hat z.B. auch Masse.

${ }^{30} \mathrm{Vgl}$. Ziel, S. 266ff, Kap. 10, Paragr. 5, zitiert in Anm. 20.

${ }^{31}$ Vgl. etwa K. Pearson, The Grammar of Science (London, 2. Auf. 1900); B. Stallo, The Concepts and Theories of Modern Physics (New York, 2. Auf. 1897); H. Poincaré, La Science et l'hypothèse (Paris, 1903).

${ }^{32}$ Ziel (Anm. 20), Kap. 10, Paragr. 2, S. 248.

${ }^{33}$ Vgl. Ziel (Anm. 20), Kap. 10, Paragr. 3, S. 249. 
an irgend einer Stelle im theoretischen Gefüge sind Duhem zufolge hinreichend, wieder Kohärenz zwischen Theorie und Erfahrung herzustellen. Es bleibt also 'dem Physiker die Sorge überlassen, unter einer Unzahl gleichgut möglicher symbolischer Übersetzungen zu wählen. Sie bedingen keineswegs eine bestimmte Wahl und sie verleihen dieser auch keine Gewißheit'. ${ }^{34}$ Dieser Unterbestimmtheit der Theorie durch Experimente mußte Duhem nun entgegnen, wenn er physikalische Theorie nicht in den Rang der Beliebigkeit abrutschen lassen wollte. Er tat dies auf zweifache Weise (unter Vorwegnahme des erst von Reichenbach explizit in die Wissenschaftstheorie eingeführten Gegensatzes von 'Entdeckungs- und Rechtfertigungszusammenhang'); ${ }^{35}$ in beiden Argumentationssträngen spielt Wissenschaftsgeschichte eine zentrale Rolle.

Betreffs der erkenntnispsychologischen Frage, wie es denn zur Aufstellung einer Hypothese in Auszeichnung vor vielen anderen denkbaren komme, sagt Duhem: 'Einzig die Intuition, die zu der Form der aufzustellenden Theorie rät, leitet diese Wahl', ${ }^{36}$ wobei es nicht nur bei dieser psychologischen Benennung bleibt, sondern das Funktionieren dieses ‘intuitiven Ersinnens' (z.B. via Analogiebildung) durch zahlreiche wissenschaftshistorische Beispiele illustriert wird. ${ }^{37}$ Wissenschaftsgeschichte hat an dieser Stelle also die Rolle von Belegmaterials (i) positiv für Duhems Erkenntnispsychologie (ii) negativ gegen den naiven Empirismus und sein Induktionskonzept. ${ }^{38}$

Die andere, wissenschaftstheoretische Frage, wie denn eine bereits (auf ' $\mathrm{krummen}$ Wegen') aufgefundene Theorie gerechtfertigt werden könne (d.h. wie ein System von Hypothesen vor anderen mit gleichem Beobachtungsgehalt ausgezeichnet werden kann), begegnet Duhem durch die These, daß es im Verlauf der historischen Entwicklung wissenschaftlicher Theorien asymptotisch eine 'naturgemäße Klassifikation' einstelle, ${ }^{39}$ d.h. daß die physikalische Theorie

zwischen den verschiedenen experimentellen Gesetzen eine logische Beziehung herstelle, die gleichsam ein Bild und ein Reflex der wirklichen Ordnung ist, in der die Realitäten, die uns entgehen, angeordnet sind. ${ }^{40}$

Die einzige Möglichkeit einer nicht-zirkulären Begründung für die Wahl einer Theorie lag für Duhem somit in dem wissenschaftshistorisch zu führenden Nachweis der Einbettung dieser Theorie in eine Tradition, als deren vorläufig willkürfreiester Endpunkt sich verstehen ließ. ${ }^{41}$ Duhems historischen Forschungen lag konsequentermaßen die These zugrunde, daß diese Annäherung der physikalischen Theorie an die strukturelle Korrespondenz der Aussagenverknüpfungen mit den

${ }^{34}$ Ziel (Anm. 20), S. 264; Kap. 10, Paragr. 5.

${ }^{35} \mathrm{Vgl}$. Bulletin (Anm. 12), Abschn. 2 (démonstration versus invention).

${ }^{36}$ Ziel (Anm. 20), S. 264; im Bulletin (Anm. 12) spricht Duhem von Wissenschaftsgeschichte als 'guide précieux pour l'inventeur qui veut ouvrir des voies nouvelles', S. 267.

${ }^{37}$ U.a. Ampère, Leonardo, Newton; siehe auch D. H. Mellors 'Models and Analogies in Science-Duhem versus Campbell?', ISIS, 59 (1968), 282-9.

${ }^{38} \mathrm{Vgl}$. zu (i) u.a. das Resumé in Duhems 'Études..., Bd. I, S. III 'nous réclamons le récit détaillé qui a conduit l'inventeur à sa découverte'; und zu (ii) Duhems Ausführungen über das 'fingierte Experiment' in Ziel (1908) (Anm. 20).

${ }^{39}$ Vgl. Ziel (Anm. 20), Kap. 3, Paragr. 1, S. 35.

${ }^{40}$ Ibid.

${ }^{41}$ Deshalb galten seine frühesten historischen Untersuchungen der Thermodynamik... Tatsächlich liegt hier die Wurzel für die Nachhaltigkeit des Interesses Duhems an Wissenschaftsgeschichte: vgl. R. N. D. Martin. 'The genesis of a mediaeval historian: Pierre Duhem and the origins of statics', Annals of Science, 33 (1976). 11929. 
kausalen Verkettungen der Sachverhalte sich ganz allmählich, in kleinsten, aufeinanderfolgenden Modifikationen vollzog (Kontinuitätsthese). ${ }^{42}$

Zusammenfassend: Duhems Konzept der Wissenschaft als bloß beschreibender Organisation von Hypothesen zu einem theoretischen System (Holismus), seine Vorstellung vom kompliziert-vermittelten Zusammenhang zwischen Experiment und Theorie (Theorienbeladenheit) sowie Tatsache und Interpretation (Konventionalismus) fixierten die Betrachtungsweise, mit der Duhem sich der Wissenschaftsgeschichte näherte (Entfaltung der Tendenz zu asymptotischer naturgemäßer Klassifikation) und sein Modell der Wissenschaftsgeschichtsentwicklung, das er in seinen Studien zu bestätigen suchte (Kontinuitätsthese).

\section{Machs historisch-kritische Darstellung}

Der Ausgangspunkt Machs ist, sehr ähnlich dem Duhems, das Verständnis wissenschaftlicher Theorien als "ökonomische Anpassung der Gedanken an die Tatsachen und der Gedanken untereinander ${ }^{43}$ (Theorieninstrumentalismus). Meine Darlegung der Vorstellungen Machs konzentriert sich im folgenden auf die Verschiedenheiten beider Ansätze.

Anders als Duhem verstand Mach wissenschaftliche Theorien als systematisierte Formen des Alltagsdenkens, aus dem diese sich durch Präzisierung allmählich abgelöst hätten ${ }^{44}$ wohingegen Mach in der Schilderung wissenschaftlicher Entwicklungen den Akzent eher auf die Ablösung durch grundlegend andere Theorien legte. Höhepunkt seiner 'historisch-kritischen[!] Darstellungen' war somit nicht der Nachweis, das eigene Auffassungen geringst mögliche Abweichungen vom historisch-verankerten Wissen bedeuteten, ${ }^{45}$ sondern umgekehrt weitreichende Kritik gerade der fundamentalsten Konzepte der historisch gefestigten Theorien (wie z.B. die am 'absoluten Raum, der absoluten Zeit und der absoluten Bewegung'. ${ }^{46}$ Anders als bei Duhem fehlt bei Mach ferner das regulative Prinzip eines Konvergierens physikalischer Theorien gegen ein 'Ziel'. Während Duhems Wissenschaftsgeschichte eine abgeschlossene Abfolge von Wiederaufnahmen unter der (konservativen) Fragehaltung: 'Was von dem, das früher gültig war, gilt heute noch?' ist, betrachtete Mach Wissenschaftsgeschichte als offene Beispielsammlung für das tatsächliche Vorgehen der Wissenschaftler, also als Materialbasis für erkenntnispsychologische und -theoretische Aufschlüsse, der er die Fragestellung: 'Was von dem, das früher für wahr und unumstösslich gehalten wurde, hat sich als voreilig, als falsch herausgestellt?' entgegenbrachte ${ }^{47}$ Dies bedeutete eine scharfe Frontstellung Machs gegen alle Varianten des (neo)kantianischen Apriorismus und Dogmatismus: alle vermeintlichen Aprioris stehen nach Mach unter dem Verdacht der Kontingenz, womit der gesamte Ansatz für Mach diskreditiert ist; weil

\footnotetext{
${ }^{42} \mathrm{Vgl}$. z.B. Duhem, Le Système du monde 10 Vols. (Paris, 1913-59), I, S. 5: 'En la genèse d'une doctrine scientifique, il n'est pas de commencement absolu; si haut que l'on remontre la lignée des pensées qui ont préparé, suggeré, annoncé cette doctrine, on parvient toujours à des opinions qui, à leur tour, ont été préparées, suggérées et annoncées; et si l'on cesse de suivre cet enchaînement d'idées qui ont procédé les unes des autres, ce n'est pas qu'on ait mis la main sur le maillon initial, mais c'est que la chaîne s'enforce et disparait dans les profondeurs d'un insondable passé; und Miller (Anm. 2), S. 227.

${ }^{43} \mathrm{Vgl}$. Mach, Erkenntnis und Irrtum (Leipzig, 1905).

${ }^{44} \mathrm{Vgl}$. hierzu insb. Machs Studie, Die Kultur der Mechanik (Stuttgart, 1915).

${ }^{45} \mathrm{Vgl}$. Duhem, Die Wandlungen der Mechanik und der modernen Naturerfahrung.

${ }^{16}$ In Machs, Mechanik (Anm. 10), Teil 7 v.Kap.II: 'Übersichtliche Kritik der Newtonschen Aufstellungen'.

${ }^{47}$ Vgl. E. N. Hiebert, Mach's philosophical use of the history of science, in: R. H. Stuewer (Hrsg.), Historical and Philosophical Perspectives of Science (Minneapolis, 1970), S. 184-203.
} 
metaphysische Setzungen in wissenschaftlichen Theorien nur dogmatisch verteidigt werden können, ohne daß sie ihrerseits wissenschaftliche gerechtfertigt werden können, plädierte Mach (hierin naiv empiristisch) für deren Elimination. ${ }^{48}$ Die besondere Bedeutung der Wissenschaftsgeschichte besteht für Mach darin, daß nur durch historische Studien diese gedanklichen Vorurteile als solche zu erkennen sind:

Schwierigkeiten erwarten uns nur, wenn wir in die Schule und in die höheren Studien eintreten, wo uns Sätze als selbstverständlich hingestellt werden, die oft eine mehrtausendjährige Gedankenarbeit gekostet haben. Auch hier gibt es nur einen Weg zur Aufklärung: Historische Studien... Wer nur eine Ansicht oder eine Form einer Ansicht kennt, glaubt nicht, dass je eine andere dagewesen, glaubt nicht, dass je eine andere kommen wird, der zweifelt nicht, der prüft nicht. ${ }^{49}$

Festzuhalten bleibt, daß Machs Erkenntnisinteresse an Wissenschaftsgeschichte ganz anders als bei Duhem gelagert und durchaus mit dem kursorischen, selektiven Zugang vereinbar war, mit dem Mach in seinen historisch-kritischen Darstellungen sich ausschließlich auf vorliegende Quellen oder Studien anderer stützend ohne eigene Quellenforschung $\mathrm{zu}$ betreiben, auswertend durchsichtete und in seinem Sinn interpretierte.

\section{Machs Duhem-Replik und Duhems Mach-Besprechung: Differenzen der Auffassung und gegenseitige Kritik}

Zusätzlich zu den im vorigen Abschnitt bereits besprochenen Differenzen gibt es bislang nicht berücksichtigte Quellen, die Aufschluß darüber geben können, wie beide Denker sich selbst voneinander abgrenzten.

\subsection{Machs Bemerkungen zu Duhem}

Vorab: es kann kein Zweifel daran bestehen, daß Mach Duhems Werk aufrichtig schätzte. Schon die freundlichen Worte des 65-jährigen Mach an Duhem wiegen viel: mir ist kein einziger Fall bekannt, in dem Mach Scheinkomplimente verteilt hätte. ${ }^{50}$ Weiterhin empfahl Mach dem Verleger Barth die Übersetzung von Duhems wissenschaftstheoretischem Hauptwerk La théorie physique: son objet et son structure und ermöglichte somit erst die breite Rezeption Duhems im deutschsprachigen Raum, insb. in Wien. ${ }^{51}$

Dennoch übersah Mach nicht, daß bei allen Parallelen auch tiefgehende Differenzen zwischen seinem und Duhems Ansatz bestanden. Mir sind zwei Quellen bekannt, aus denen deutlich wird, wie Mach sich von Duhem abgrenzte; zum einen

\footnotetext{
${ }^{48}$ Mach war sich nicht im Klaren darüber, wie weit dieser 'metaphysischen Wurzeln' ins EingemachtPhysikalische hineinwachsen: die theologische Verquickung, in der Newtons Mechanik und die Cambridger Theologie um Henry More stehen, wurde erst durch Cassirer (1910ff.), Burtt (1926) und Koyré (1939ff.) allgemein bekannt.

${ }^{49}$ E. Mach, Die Geschichte und die Wurzel des Satzes von der Erhaltung der Arbeit (Prag, 1872), 2. Auflage 1909; S. 1ff.

${ }^{50}$ Hingegen zeigt Machs Korrespondenz, daß er sehr an dem Gedankenaustausch mit ihm nahestehenden Denkern interessiert war; vgl. die Korrespondenzen mit Avenarius, Schuppe, Ziehen, Pearson u. Stallo in: J. Thiele (Hrsg.), Wissenschaftiche Kommunikation. Die Korrespondenz Ernst Machs (Kastellaun, 1978), und die Korrespondenz Machs mit Adler, Dingler, Höffding, Petzoldt u. Thomsen in: J. T. Blackmore u. K. Hentschel (Hrsg.), Ernst Mach als Aussenseiter. Machs Briefwechsel über Philosophie und Relativitätstheorie mit Persönlichkeiten seiner Zeit (Wien, 1985) [im folgenden zitiert als Blackmore/Hentschel].

${ }_{51}$ Vgl. z.B. L. Schäfer im Reprint von Ziel (Anm. 20), S. xv.
} 
Passagen aus späteren Auflagen seiner Mechanik, zum anderen ein Brief Machs an Friedrich Adler.

Zunächst zu ersterem: Im 'Zusatz 2' der 6. Auflage seiner Mechanik bespricht Mach Duhems Kontinuitätsthese (vgl. Abschnitt 4), derzufolge (in Machs Worten): 'die wissenschaftlichen Gedanken der Renaissance...durch eine sehr langsame, allmähliche Entwickelung in kleinen Schritten aus jenen des griechischen Altertums hervorgegangen [sind.]' Konträr zu Duhems Akzentuierung der (positiven) Vorläuferrelationen, die Duhem aufzuspüren bemüht war, betont Mach das (negative) kritische Moment der Korrektur, der Überwindung von Fehleinsichten und der begrifflichen Differenzierung, verstanden als Abgehen von uneindeutigen, fehlleitenden Termini: ${ }^{52}$

Bei Abschätzung der Bedeutung eines Forschers wird es also wohl nur darauf ankommen, welchen neuen Gebrauch derselbe von alten Einsichten gemacht hat, und unter welcher Opposition der Zeitgenossen und Nachfolger seine Einsichten zur Geltung gelangt sind. Von diesem Standpunkt betrachtet, scheint mir Duhem in seiner Pietät gegen Aristoteles doch etwas zu weit zu gehen.

Am Ende von 'Zusatz 5' findet sich ein Hinweis auf die zweite wichtige Differenz, die Mach bemerkte: für letzteren war.das Endziel der Mechanik die vollständige und 'ökonomische Anpassung der Gedanken (Theorien) an die Tatsachen (Daten)', während Duhem vom Endstadium der Theorie, gegen das diese seiner Überzeugung zufolge asymptotisch konvergiere, eine 'naturgemäße Klassifikation', also eine Art struktureller Korrespondenz zwischen Zeichensystem (Theorie) und Bezeichnetem (Natur) erwartete. Diese verschiedenartigen Vorstellungen vom Endstadium der Naturwissenschaft in the long run haben wiederum tiefe Wurzeln in ontologischen Prämissen beider Denker. Für den Phänomenalisten Mach konnte Theorie nie mehr als nützliches Instrument zur ökonomischen Erfassung der Verkettung von 'Elementen' ( elementaren Sinnesempfindungen) sein (Theorieninstrumentalismus). Demgegenüber verlangte Duhem von einer reifen Theorie Aufschluß über die reale Struktur des Wirklichen (asymptotischer Theorienrealismus).

Die offenste Einschätzung Duhems durch Mach erfahren wir aus einem Brief Machs an den Machianer und Übersetzer von Duhems 'Ziel und Struktur' (Anm. 20) Friedrich Adler vom 22. April 1908: ${ }^{53}$

Mit dem Grade der Uebereinstimmung mit Duhem bin ich vorläufig ganz zufrieden. Bei der Macht und dem EinfluB, den die Scholastik und der Katholizismus noch immer in Frankreich hat, ist es immerhin möglich, dass Duhem irgend einen verborgenen Teufel im Hintergrund nährt; ist er doch ein Verehrer des Thomas von Aquin, woraus er gar kein Hehl macht. Aber was liegt daran, so lange er den Teufel nicht los lässt. Vielleicht will er die Physik nur von der Metaphysik befreien, um für letztere der Physik gegenüber Spielraum zu gewinnen. Mit der Metaphysik sollen Philosophen und Theologen treiben, was sie wollen. Wenn dafür die Physiker, Physiologen und Psychologen sich gewöhnen ohne Metaphysik auszukommen, so ist alles gewonnen. Die Zustimmung eines Physikers wie Duhem auf physikalischem Gebiet ist sehr zu schätzen; seine unfassbare Metaphysik braucht uns nicht zu geniren; warten wird, bis sie fassbar wird.

\footnotetext{
52 'Mechanik' (Anm. 10), S. 563.

${ }^{53}$ Vollständig wiedergegeben in Blackmore/Hentschel (Anm. 50), S. 50.
} 
Faßbarer als in Duhems Ausführungen über naturgemäße Klassifikation und seinen 'Bekenntnissen' wurde seine Metaphysik jedoch nicht mehr. Diese abwartende Haltung Machs erklärt auch, warum nach 1908 nur noch einmal eine kurze Nachricht von ihm Duhem erreichte. Beide Denker verstarben 1916, fern von den intellektuellen Zentren ihrer Wirkungskreise in persönlicher Isolation.

\subsection{Duhem über Mach}

Duhem äußerte sich zu Mach in vereinzelten Passagen seines wissenschaftstheoretischen Hauptwerkes und ausführlich in der erwähnten Rezension der Machschen Mechanik. Aus dieser Quelle ist zunächst entnehmbar, daß Duhem Machs wissenschaftshistorische Ausführungen sehr wohl im Zusammenhang mit Machs methodologischen Ausführungen gesehen hat. Ich referiere hier nur die Punkte, in denen Duhem Abweichung feststellte.

Im Abschnitt 4 seiner Besprechung wirft Duhem Mach vor, daß dieser Wissenschaftsgeschichte nur als Mittel betrachte, um seine wissenschaftspsychologischen und -theoretischen Vorstellungen $\mathrm{zu}$ illustrieren, ohne eigene Forschungen zu betreiben: 'Dans la longue suite des transformations, qui composent un tel progrès, $M$. Mach choisit seulement ce qui aide à comprendre le plan définitif.' Dieses projektive Auswählen mache Geschichte $\mathrm{zu}$ geradlinig und führe dazu, daß einige Gebiete zu Unrecht völlig unter den Tisch fielen: 'il abrège l'exposé des débuts trop anciens et trop confus pour que la Science actuelle en ait gardé la marque'. Machs Zugang sei mehr der eines Logikers denn der eines Psychologen:

les tableaux historiques tracés par M.E. Mach [sont] trop simples, trop clairs, trop parfaitement ordonnés; l'évolution, telle que ces tableaux la retracent, tend trop constamment, trop surement au but qu'elle doit atteindre; la marche de l'esprit humaine a été en réalité, plus hésitante, plus tâtonnante'. ${ }^{54}$

Am Beispiel Descartes zeigt Duhem dann im einzelnen auf, wie verkürzt die Darstellung Machs mitunter ist. Schwerwiegend ist jedoch, daß ausgerechnet Mach, der immer beansprucht hat, den tatsächlichen Verlauf von Wissenschaft im Blick zu haben, von Duhem vorgeworfen wurde, mit getrübtem Auge zu sehen.

Ungeachtet dieser Kritik an Machs Zugang zur Wissenschaftsgeschichte, die dieser mit veränderten Vorzeichen auch Duhem gegenüber hätte äußern können, sieht Duhem in der Absicht, die hinter Machs Programm steht, durchaus einen Schritt in Richtung auf eine 'physique homogène', eine 'physique comparée' im Rahmen des Programms, das Duhem 'Energetik' und Mach 'neutralen Monismus' nannten. ${ }^{55}$

\section{Zusammenfassende kritische Bewertung beider Auffassungen}

Die wissenschaftsgeschichtlichen Schriften beider demonstrieren den Effekt, den ein präsupponiertes Modell der Wissenschaftsgeschichtsentwicklung in Verbindung mit weiteren 'tacit assumptions' ${ }^{56}$ auf die historische Darstellung hat. Nicht umsonst stand genau dies immer im Zentrum der Kritik durch Zeitgenossen u. Nachfolger. ${ }^{57}$

\footnotetext{
${ }^{54} \mathrm{Vgl}$. Duhem, Bulletin (Anm. 12), S. $267 \mathrm{ff}$.

${ }^{55}$ Vgl. die Korrespondenz Mach-Ostwald zur Abgrenzung Machs von der Energetik Ostwald-HelmDuhemscher Prägung.

${ }^{56}$ Anspielung auf M. Polanyi, Personal Knowledge (London, 3. Aufl. 1969).

${ }^{57}$ Finen Überblick gibt Jaki im Vorwort zur englischen Übersetzung von Duhem, To Save the Phenemena (Chicago und London, 1969), S. xix; vgl, auch Anm. 67 f.
} 
Duhems Kontinuitätsthese, die ihn zur Sichtung der bis dahin nicht beachteten mittelalterlichen Dokumente in der Bibliothèque Nationale motiviert hatte und damit unstrittig von enormen heuristischen Wert für die Emanzipation der Wissenschaftsgeschichte vom Dogma des 'finsteren Mittelalters' gewesen ist, diese Kontinuitätsthese war es auch, die ihn z.B. die Bedeutung Jordanus de Nemores als Vorläufer von Leonardo ${ }^{58}$ oder z.B. die Nicole d'Oresmes als Vorläufers der analytischen Geometrie und der modernen Kosmologie ${ }^{59}$ überschätzen ließ. Bei der Überschätzung der Bedeutung der Pariser Schule in der Nachfolge Buridans für die Herausbildung der mechanischen Grundprinzipien mag neben dieser Modellbeladenheit auch der ausgeprägte Chauvinismus und Katholizismus Duhems mitgespielt haben. ${ }^{60}$

Machs pädagogisch-logische Absicht der Verdeutlichung der gegenwärtigen Konzepte durch deren Herausbildung, Durchsetzung und Entwicklung führte ihn zwangsläufig zum Übersehen all dessen, was aus heutiger fachwissenschaftlicher Sicht irrelevant ist. So liegt es nahe, sich mit Duhem über den Umstand zu wundern, daß der Naturkonzeption des Mittelalters in Machs 'historisch-kritischen Darstellungen' nur verschwindend wenig Raum gegeben wird. ${ }^{61}$ Wenngleich Duhem in seinen chauvinistischen Schriften und Vorträgen aus den Jahren des 1. Weltkrieges Mach nicht ausdrücklich erwähnte, dürfte er doch auch an ihn gedacht haben, als er der deutschen Geschichtsschreibung die Tendenz unterstellte, selektive Auswahl nur der Fakten $\mathrm{zu}$ betreiben, die ein vorausgesetztes Bild bestätigen und andere Fakten umbiegen $\mathrm{zu}$ wollen. ${ }^{62}$

In beider Schriften wurde also der 'Glanz' der Geschlossenheit der Darstellung und des widerspruchsfreien sich-Einfügens in das von ihnen entworfene Bild der wissenschaftsgeschichtlichen Entwicklung erreicht durch das 'Elend' der 'Modellbeladenheit' ihrer Entwürfe mit der Gefahr der verzerrten Darstellung in Einzelheiten. Auch in der heutigen Wissenschaftsgeschichtsschreibung ist dies durchaus beobachtbar. ${ }^{63}$ Dies ist u.a. darauf zurückführbar, daß die Wissenschaftshistoriker wie schon Duhem und Mach meist ausgebildete Fachwissenschaftler sind, denen die Paradigmen heutigen naturwissenschaftlichen Vorgehens 'natürlich' auch die Brille für die Betrachtung historischer Quellen

\footnotetext{
${ }^{58} \mathrm{Vgl}$. hierzu schon der oben zitierte Einwand Machs im 'Zusatz 5' der 6. Auflage der Mechanik (Anm. 10), 1908 , S. 566.

${ }^{59}$ Vgl. dazu E. Grant, 'Pierre Duhem on Oresme and Celestial incommensurability', in E. Grant (Hrsg.), Nicole Oresme and the Kinematics of Circular Motion (Madison, 1971), S. 378-82.

${ }^{60} \mathrm{Vgl}$. H. W. Paul, 'Pierre Duhem: the scientific philosophy of a modern believer', in H. W. Paul (Hrsg.), The Edge of Contingency (Gainesville, 1979), S. 137-78.

${ }^{61}$ In seiner Mechanik (Anm. 10) springt Mach etwa bei der Darstellung der Entwicklung des Hebelgesetzes von Archimedes gleich zu Leonardo da Vinci; vgl. hingegen die detailliertere story in Duhems Origines (Anm. 21).

${ }_{62}^{2}$ P. Duhem, 'Les Sciences historiques', in La Science Allemande (Paris, 1915), S. 53-72; vgl. z.B. H. W. Paul, 'Science and the historian's craft', Journal of the History of Ideas, 33 (1072), 497-512; insb. $508 \mathrm{f}$.

${ }^{63}$ Eine Einschätzung der positiven Bedeutung dieses engen Nexus zwischen Wissenschaftsgeschichte und Wissenschaftstheorie für letztere gibt K. Hübner, 'Duhems historische Wissenschaftstheorie und ihre gegenwärtige Weiterentwicklung', Philosophia Naturalis, 13 (1971), 81-97; vgl. auch Ph. L. Quinn, 'What Duhem really meant', Boston Studies in the Philosophy of Science, 14 (1978), 33-56; eine wissenschaftstheoretische Kritik einzelner Thesen Duhems unternimmt R. M. Yoshida, 'Five Duhemian Theses', Philosophy of Science, 42 (1975), $29-45$.
} 
abgeben. ${ }^{64}$ Diese These bestätigt sich durch den Vergleich der Arbeiten dieser Gruppe von Historikern mit denen soziologischer Provenienz. ${ }^{65}$

Duhem zog 1915 interessante Parallelen zwischen 'Dokument und Experiment'; 'Wissenschaftsgeschichte und Theorie': wie Theorien nämlich am Experiment getestet würden, ${ }^{66}$ so müssten auch Leitmotive historischer Forschung 'getestet' werden, und zwar am neuen, bislang unbekannten oder vergessenen Dokument. Durch seine eigenen Arbeiten ist Duhem dies in betreff der Einschätzung des Mittelalters hervorragend gelungen, wenngleich er sich gefallen lassen mußte, daß jüngere Historiker/innen (z.B. Anneliese Maier oder Helène Metzger-Bruhl) auch die von ihm modellierten Ansichten 'testeten' und in Einzelfällen revidierten. ${ }^{67}$ Der Autor schließt sich Imre Lakatos an, wenn dieser feststellt: 'Geschichte ohne theoretische Vorurteile ist unmöglich'. ${ }^{68}$

Ein Ausweg aus dieser Sackgasse der Abfolge von jeweils einseitig tendenziösen Studien wäre, meine ich, das Bemühen der Wissenschaftshistoriker (egal welcher Herkunft), historische Studien vor dem Hintergrund eines Pluralismus von Modellen der Wissenschaftsgeschichtsentwicklung ${ }^{69}$ erstellen, d.h. im Einzelfall zu prüfen: welches der bekannten Modelle bewährt sich, welche Umstände werden jeweils 'bedeutsam' oder auch: 'Wie müssen die vorliegenden Modelle verfeinert werden, um dem jeweiligen Fall gerecht zu werden', ${ }^{70}$ Aus dieser engeren Verbindung von Wissenschaftsgeschichte mit Wissenschaftstheorie würde auch eine qualitative Verbesserung beider resultieren

${ }^{64} \mathrm{Vgl}$. z.B. den Streit zwischen I. Szabo und A. Hermann anläßlich des Erscheinens der Geschichte der mechanischen Prinzipien des ersteren; siehe Vorwort von A. Hermann zur Sonderausgabe der Stiftung Volkswagenwerk (Basel und Stuttgart, 1976), S. xi-xix u. I. Szabo, 'Bemerkungen zur Literatur über die Geschichte der Mechanik', Humanismus und Technik, 21/22 (1977/78), 121-54.

${ }^{65}$ Frühe Beispiele sind: W. Jerusalem, 'Die soziologische Bedingtheit des Denkens und der Denkformen' in M. Scheler (Hrsg.), Versuche zu einer Soziologie des Wissens (München und Leipzig, 1924), S. 182-207; L. Fleck, Entstehung und Entwicklung einer wissenschaftlichen Tat sache [1935], Reprint mit Einleitung von L. Schäfer und L. Schnelle (Frankfurt, 1980), und E. Zilsels Studien Ǔber die sozialen Ursprünge der neuzeitlichen Wissenschaft, mit Einl. herausgegeg. v. W. Krohn (Frankfurt, 1976); neuere Beiträge sind der Diskussion von T. S. Kuhn Die Struktur von wissenschaftlicher Revolutionen [1962] entsprungen: siehe die Literaturübersicht von W. Kaminsky im Philosophischen Literaturanzeiger, 1978.

${ }^{66}$ Und zwar ganzheitlich, nicht einzelne Theoreme; s.o. zu 'experimentum crucis'.

${ }^{67} \mathrm{Vgl}$. die Beträge zur Gedächtnissitzung der 'Groupe français adhérant à l'académie Internationale d'histoire des sciences am 27. Januar 1937, abgedruckt in Archeion [Rom], 19 (1937), 121-51. Neue Beispiele für Kritik an einzelnen wissenschaftlichen Thesen Duhems sind: O. Neugebauer, The Exact Sciences in Antiquity (Providence, 2. Aufl. 1957), S. 206: 'Duhems total ignorance of Ptolemy's lunar theory is a good example of a rapid decline of the history of science'; M. Clavelin, 'Galilei et le refus de l'équivalence des hypothèses', Revue d'histoire des sciences, 17 (1964), 305-30, insb. S. 308: 'Cette interpretation paradoxale, veritable rehabilitation de la science traditionelle à été soutenue, on le sait, par Pierre Duhem, qui, s'autorisant de la science moderne et de ses derniers développements, jugeait radicalement erroné la position de Galilei'; R. Palter, 'An approach to the history of early astronomy', Studies in the History and Philosophy of Science, 1 (1970), 93-133, insb. S. 130, a. 38: 'Duhem's entire historical analysis is subtly distorted by his own philosophical prejudices'.

${ }^{68}$ Imre Lakatos, 'Die Geschichte der Wissenschaft und ihre rationalen Rekonstruktionen', in W. Diederich (Hrsg.), Theorien der Wissenschaftsgeschichte (Frankfurt, 1978), S. 53-120, Zitat S. 85 f; vgl. auch K. R. Popper, Das Elend des Historizismus (Tübingen, 5. Aufl. 1979), Abschnitt 31, S. 115 ff. Machs wie Duhems Wissenschafts 'geschichten' sind ausgezeichnete Beispiele für planvoll zusammengestellte 'Ereignisse, die in normativer Weise ausgewählt und interpretiert werden' (ibid., S. 86f.).

${ }^{69} \mathrm{Vgl}$. die Übersichten in W. Diederich (Anm. 68) und in R. Stuewer (Hrsg.), Historical and Philosophical Perspectives of Science (Minneapolis, 1970).

${ }^{70}$ Ansätze hierzu sind z.B. A. Ströker, Theoriewandel in der Wissenschaftsgeschichte. Chemie im 18. Jahrhundert (Frankfurt, 1982); W. Kaiser, Über die rationale Rekonstruierbarkeit theoretischer Entwicklungen in der Elektrodynamik des 19. Jahrhunderts (Stuttgarter Phil. Diss., 1975), und M. Wolff, Geschichte der Impetustheorie. Untersuchungen zum Ursprung der klassischen Mechanik (Frankfurt, 1978). 
gemäß der Lakatos'schen Paraphrase der berühmten Kantischen Verschränkung von Sinnlichkeit und Verstand: ${ }^{71}$

W issenschaftsgeschichte ohne W issenschaftsphilosophie ist blind; Wissenschaftsphilosophie ohne Wissenschaftsgeschichte ist leer.

\section{Danksagungen}

Die fünf Briefe Machs an Duhem verwahren neben anderen Briefen aus Duhems Nachlaß die Archives de l'Académie des Sciences, Paris; Duhems acht Briefe an Mach liegen im Ernst Mach Institut der Fraunhofer-Gesellschaft in Freiburg im Breisgau, das einen Großteil des Mach-Nachlasses verwaltet. Beiden Stellen sei gedankt für die Genehmigung zur Veröffentlichung der Briefe, die in diesem Aufsatz erstmals und vollständig abgedruckt werden.

Ferner danke ich den Herren Prof. Lothar Schäfer (Philosophisches Seminar der Universität Hamburg) und Prof. Andreas Kleinert (Institut für Geschichte der Naturwissenschaften, Hamburg) für Hinweise zu Person und Werk Duhems. Von Herrn Dr. John T. Blackmore (z.Z. Japan) empfing ich viele Anregungen zu Ernst Mach; Herrn Prof. Don Howard (USA) danke ich herzlich für wertvolle Hinweise zu Duhem und dem wissenschaftsphilosophischen Umfeld.

Nach Fertigstellung des Manuskriptes erfuhr ich von Herrn Dr. Michel Paty (Paris), den ich im Bostoner Forschungsarchiv der Collected Papers of Albert Einstein traf, von dessen Studie über 'Mach et Duhem'. ${ }^{72}$ Ihm sei gedankt für den freundlichen Hinweis auf einige Transkriptionsfehler in meiner Abschrift der von ihm nicht berücksichtigten Korrespondenz.

\footnotetext{
${ }^{71}$ Lakatos (Anm. 68), Zitat S. 56.

${ }^{72}$ M. Paty, 'Mach et Duhem. L’Épistémologie de "savant-philosophes"', in Épistémologie et Matérialisme. Seminaire sous la direction de Olivier Bloch (Paris, 1986), S. 177-218.
} 